NOTICE: this is the author's version of a work that was accepted for publication in Automatica. Changes resulting from the publishing process, such as peer review, editing, corrections, structural formatting, and other quality control mechanisms may not be reflected in this document. Changes may have been made to this work since it was submitted for publication. A definitive version was subsequently published in Automatica, Vol. 48, no.7 (2012). DOI: 10.1016/j.automatica.2012.04.005. 


\title{
Formation Control of Multiple Elliptical Agents with Limited Sensing Ranges
}

\author{
K. D. Do \\ School of Mechanical Engineering, The University of Western Australia \\ 35 Stirling Highway, Crawley, WA 6009, Australia
}

\begin{abstract}
This paper presents a design of cooperative controllers that force a group of $N$ mobile agents with an elliptical shape and with limited sensing ranges to perform a desired formation. The controllers guarantee no collisions between any agents in the group. The desired formation can be stabilized at feasible reference trajectories with bounded time derivatives. The formation control design is based on an algebraic separation condition between ellipses, Lyapunov's method, and smooth or $p$-times differentiable step functions. These functions are introduced and incorporated into novel potential functions to solve the collision avoidance problem without the need of switchings under the agents' limited sensing ranges.
\end{abstract}

Keywords: Formation control, elliptical agents, collision avoidance, potential functions, Lyapunov's method

\section{Introduction}

Various methods have been proposed for formation control of multiple agents. Here, three popular methods are briefly mentioned. The leader-follower method (e.g., Wang (1991), Das et al. (2002), Hu and Feng (2010)) uses several agents as leaders and others as followers. This method is easy to understand and ensures formation maintenance if the leaders are disturbed. However, the desired formation cannot be maintained if followers are perturbed unless a formation feedback is implemented, Egerstedt and $\mathrm{Hu}$ (2001). The behavioral method (e.g., Balch and Arkin (1998), Jonathan et al. (2003)), where each agent locally reacts to actions of its neighbors, is suitable for decentralized control but is difficult in control design and stability analysis since group behavior cannot explicitly be defined. The virtual structure method (e.g., Ogren et al. (2004), Tanner and Kumar (2005), Do (2007)) treats all agents as a single entity. This method is amenable to mathematical analysis but is difficult to deal with time-varying formation structure.

Research works on formation control usually utilize one or more of the above methods in a centralized or a decentralized manner. Centralized strategies (e.g., Rimon and Koditschek (1992), Egerstedt and $\mathrm{Hu}$ (2001)) use a single controller that generates collision free trajectories in the workspace. These strategies guarantee a complete solution but require high computational power and are not robust. Decentralized schemes (e.g., Stipanovic et al. (2004), Olfati-Saber (2006), Dimarogonas et al. (2006), Do (2007), Hussein and Stipanovic (2007), Hussein and Bloch (2008)) require less computational effort but have difficulties in controlling critical points, especially when collision avoidance between the agents is a must.

In the above works, the agent is considered as a single point or has a circular/spherical shape. In practice, many agents have a long and narrow shape. Fitting these agents to circular disks results in a problem of the large conservative area. Let us

Email address: duc@mech.uwa.edu.au (K. D. Do) look at an example of fitting a rectangular agent with a width of $W$ and a length of $L$ to an elliptical disk with semi-axes of $a$ and $b$, and a circular disk with a radius of $r_{c}$ as shown Fig.1. A calculation shows that $a=$ $\frac{\sqrt{2}}{2} W, b=\frac{\sqrt{2}}{2} L$, and $r_{c}=$ $\frac{1}{2} \sqrt{W^{2}+L^{2}}$. Therefore, the conservative area $A_{\text {con }}$ defined as the difference between the areas enclosed by the circle and the ellipse is given by $A_{c o n}=\pi r_{c}^{2}-\pi a b=$ $\frac{\pi}{4}(L-W)^{2}$. This calculation indicates that it is much more efficient to use an el-

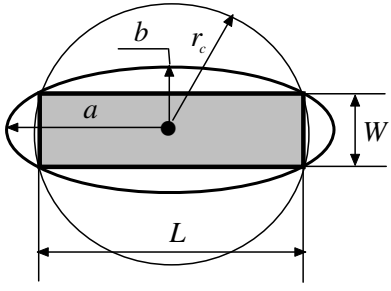

Figure 1: Fitting a rectangular agent to an ellipse and a circle lipse to bound an agent with a long and narrow shape for formation control. The above illustration motivates contributions of this paper.

This paper continues the author's previous work in Do (2007) to study a problem of designing a formation tracking system for multiple agents with an elliptical shape and limited sensing ranges. In comparison with the work in Do (2007), the formation control design and stability analysis for elliptical agents are far more complicated due to two folds. The first difficulty is that a collision avoidance condition for elliptical agents depends on both position and orientation of the agents in a complex manner. The second difficulty is that the tracking issue causes difficulties in designing a cooperative controller to guarantee negative definite of derivative of a proper potential function. To solve the aforementioned formation problem, a condition, which is applicable for formation control, for collision avoidance between the agents is derived, see Lemma 2.1. New potential functions are then proposed for the control design, see Section 4. Lyapunov's method and Chetaev's Theorem are used for the stability analysis, see Appendix B.

Due to the aforementioned difficulties in the formation control design and stability analysis, the agents considered in this paper are assumed to be fully actuated. Theoretical development in this paper sets a foundation, which can be used to 
design a formation control system for various agents with a long and narrow shape in practice. For such an application, the reader is referred to Do (2011), where a combination of the development in this paper and several control techniques in Do and Pan (2009) and Do (2010) is presented to design a formation control system for multiple underactuated surface ships with an elliptical shape.

\section{Preliminaries}

\subsection{Separation condition between two elliptical disks}

In Choi et al. (2006), the authors proposed a collisiondetection algorithm for two elliptical disks based on the discriminant of their characteristic third-order polynomial. However, calculation of the discriminant is too complicated for an application in formation control. This is because coefficients of the characteristic polynomial are complex expressions of a product of the fourth order of the position and the eighth order of cosine and sine of heading angles of the two elliptical disks. The condition, for which the two elliptical disks' discriminant is positive, can be embedded in a potential function for a formation control design despite of the discriminant's complexity, but it is problematic in stability analysis of the resulting closed loop system. It does not seem to be able to prove that undesired critical points of the closed loop system are saddle/unstable.

We here present a simpler condition for separation of two elliptical disks. This condition will be used as a condition for collision avoidance in the control design later. As such, we consider two elliptical disks $i$ and $j$ shown in Fig. 2, where $\left(x_{i}, y_{i}\right)$ denote the position of the center $O_{i}$ and $\phi_{i}$ is the heading angle of the disk $i$. These notations are similar for the disk $j$. Moreover, $\left(a_{i}, b_{i}\right)$ and $\left(a_{j}, b_{j}\right)$ denote the semi-axes of the disks $i$ and $j$, respectively.

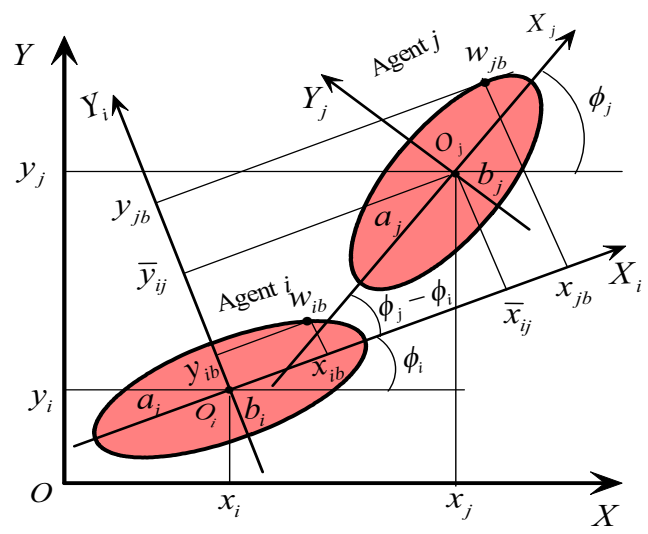

Figure 2: An agent's shape and its coordinates with respect to the earth-fixed frame $O X Y$

Lemma 2.1. Consider two elliptical disks $i$ and $j$ with the heading angles of $\phi_{i}$ and $\phi_{j}$ centered at $\left(x_{i}, y_{i}\right)$ and $\left(x_{j}, y_{j}\right)$, respectively. Let

$$
\Delta_{i j}=\frac{1}{2}\left[\left(\frac{\kappa_{i j} \hat{x}_{i j}}{\kappa_{i j}+\hat{a}_{j}^{2}}\right)^{2}+\left(\frac{\kappa_{i j} \hat{y}_{i j}}{\kappa_{i j}+\hat{b}_{j}^{2}}\right)^{2}-1\right],
$$

where $\kappa_{i j}$ is the largest solution of the following equation

$$
\left(\frac{\hat{a}_{j} \hat{x}_{i j}}{\kappa_{i j}+\hat{a}_{j}^{2}}\right)^{2}+\left(\frac{\hat{b}_{j} \hat{y}_{i j}}{\kappa_{i j}+\hat{b}_{j}^{2}}\right)^{2}-1=0,
$$

and

$$
\begin{aligned}
& \hat{a}_{j}=\frac{1}{\sqrt{T_{a}}}, \hat{b}_{j}=\frac{1}{\sqrt{T_{b}}}, \\
& {\left[\begin{array}{c}
\hat{x}_{i j} \\
\hat{y}_{i j}
\end{array}\right]=\left[\begin{array}{cc}
-\frac{1}{a_{i}} \cos \left(\alpha_{i j}\right) & -\frac{1}{b_{i}} \sin \left(\alpha_{i j}\right) \\
\frac{1}{a_{i}} \sin \left(\alpha_{i j}\right) & -\frac{1}{b_{i}} \cos \left(\alpha_{i j}\right)
\end{array}\right] \overline{\boldsymbol{p}}_{i j},} \\
& \alpha_{i j}=2 \arctan \left(\frac{2\left(T_{11} T_{12}+T_{21} T_{22}\right)}{T_{11}^{2}+T_{21}^{2}-T_{12}^{2}-T_{22}^{2}}\right),
\end{aligned}
$$

with

$$
\begin{aligned}
& T_{a}=\left(T_{11}^{2}+T_{21}^{2}\right) \cos ^{2}\left(\alpha_{i j}\right)+\left(T_{11} T_{12}+T_{21} T_{22}\right) \times \\
& \sin \left(2 \alpha_{i j}\right)+\left(T_{12}^{2}+T_{22}^{2}\right) \sin ^{2}\left(\alpha_{i j}\right), \\
& T_{b}=\left(T_{11}^{2}+T_{21}^{2}\right) \sin ^{2}\left(\alpha_{i j}\right)-\left(T_{11} T_{12}+T_{21} T_{22}\right) \times \\
& \sin \left(2 \alpha_{i j}\right)+\left(T_{12}^{2}+T_{22}^{2}\right) \cos ^{2}\left(\alpha_{i j}\right), \\
& T_{11}= \frac{a_{i}}{a_{j}} \cos \left(\phi_{i j}\right), \quad T_{12}=-\frac{b_{i}}{a_{j}} \sin \left(\phi_{i j}\right), \\
& T_{21}= \frac{a_{i}}{b_{j}} \sin \left(\phi_{i j}\right), \quad T_{22}=\frac{b_{i}}{b_{j}} \cos \left(\phi_{i j}\right), \\
& x_{i j}=x_{i}-x_{j}, \quad y_{i j}=y_{i}-y_{j}, \phi_{i j}=\phi_{i}-\phi_{j}, \\
& {\left[\bar{x}_{i j}, \bar{y}_{i j}\right]^{T}=\boldsymbol{R}_{i}\left[x_{i j}, y_{i j}\right]^{T}, }
\end{aligned}
$$

where $\boldsymbol{R}_{i}=-\boldsymbol{R}^{-1}\left(\phi_{i}\right)$ with $\boldsymbol{R}(\bullet)$ the rotational matrix. The two elliptical disks are externally separated if

$$
\Delta_{i j} \geq \delta_{i j}
$$

with $\delta_{i j}$ being a positive constant.

\section{Proof. See Appendix A.}

\section{2. $p$-times differentiable functions}

This section presents a construction of $p$-times differentiable or smooth step functions. These functions are to be embedded into a potential function to avoid discontinuities in the control law due to the agents' limited sensing ranges.

Definition 2.1. A scalar function $h(x, a, b)$ is said to be a ptimes differentiable step function if it possesses the properties:
1) $h(x, a, b)=0, \forall-\infty<x \leq a$,
2) $h(x, a, b)=1, \forall b \leq x<\infty$,
3) $0<h(x, a, b)<1, \forall x \in(a, b)$,
4) $h(x, a, b)$ is $p$ times differentiable,

where $p$ is a positive integer, $x \in \mathbb{R}$, and $a$ and $b$ are constants such that $a<b$. Moreover, if the function $h(x, a, b)$ is infinite times differentiable with respect to $x$, then it is said to be a smooth step function.

Lemma 2.2. Let the scalar function $h(x, a, b)$ be defined as

$$
h(x, a, b)=\frac{\int_{a}^{x} f(\tau-a) f(b-\tau) d \tau}{\int_{a}^{b} f(\tau-a) f(b-\tau) d \tau},
$$

with $a$ and $b$ being constants such that $a<b$, and the function $f(y)$ being defined as follows

$$
f(y)=0 \text { if } y \leq 0 \text {, and } f(y)=g(y) \text { if } y>0,
$$

where the function $g(y)$ has the following properties

a) $g(\tau-a) g(b-\tau)>0, \quad \forall \tau \in(a, b)$,

b) $g(y)$ is $p$-times differentiable,

c) $\lim _{y \rightarrow 0^{+}} \frac{\partial^{k} g(y)}{\partial y^{k}}=0, k=1, \ldots, p-1$, 
with $p$ being a positive integer. Then $h(x, a, b)$ is a p-times differentiable step function. Moreover, if $g(y)$ in (8) is replaced by $g(y)=e^{-1 / y}$ then $h(x, a, b)$ is a smooth step function.

Proof. See Do (2009).

\section{Problem statement}

\subsection{Agent dynamics}

As mentioned before this paper mainly focuses on difficulties caused by the elliptical shape of the agents in the formation control, we therefore assume that each elliptical agent $i$ has the dynamics:

$$
\dot{\boldsymbol{q}}_{i}=\boldsymbol{u}_{i}
$$

for all $i \in \mathbb{N}$ with $\mathbb{N}$ the set of all agents in the group. The vector $\boldsymbol{u}_{i}=\left[\begin{array}{lll}u_{x i} & u_{y i} & u_{\phi i}\end{array}\right]^{T}$ denotes the control input, the vector $\boldsymbol{q}_{i}=\left[\begin{array}{lll}x_{i} & y_{i} & \phi_{i}\end{array}\right]^{T}$ denotes position and orientation of the agent $i$, see Fig. 2.

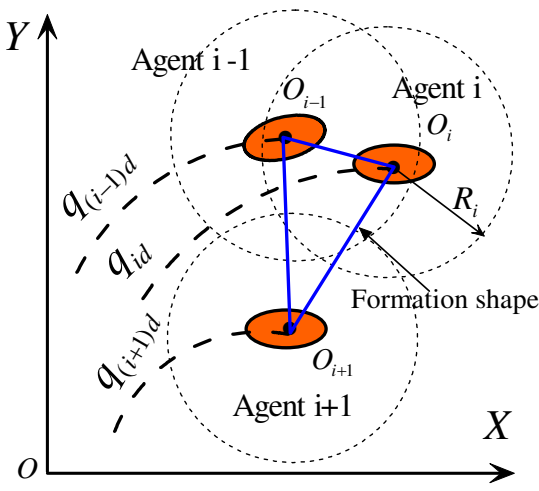

Figure 3: Formation setup.

\subsection{Formation control objective}

We first impose the following assumption on the communication and initial conditions between the agents in the group.

\section{Assumption 3.1.}

1) All the reference trajectories, $\boldsymbol{q}_{i d}\left(s_{i d}\right)=$ $\left[x_{i d}\left(s_{i d}\right) y_{i d}\left(s_{i d}\right) \phi_{i d}\left(s_{i d}\right)\right]^{T}, i \in \mathbb{N}$ with $s_{i d}$ the parameter of $\boldsymbol{q}_{i d}\left(s_{i d}\right)$, satisfy the condition:

$$
\Delta_{i j d} \geq \delta_{i j d},
$$

where $\delta_{i j d}$ is a positive constant. The function $\Delta_{i j d}$ is given in (1) with $\boldsymbol{q}_{i}$ and $\boldsymbol{q}_{j}$ replaced by $\boldsymbol{q}_{i d}$ and $\boldsymbol{q}_{j d}$, respectively, and $s_{i d}=s_{j d}$. Moreover, $\left\|\dot{\boldsymbol{q}}_{i d}\right\|$ is bounded for bounded $\left|\dot{s}_{i d}\right|$, and $\left\|\boldsymbol{q}_{i d}-\boldsymbol{q}_{j d}\right\|$ is also bounded for bounded $\left|s_{i d}\right|$ and $\left|s_{j d}\right|$.

2) The agents $i$ and $j$ have circular communication areas, which are centered at $O_{i}$ and $O_{j}$, and have radii of $R_{i}$ and $R_{j}$, see Fig. 3. The radii $R_{i}$ and $R_{j}$ satisfy the condition:

$$
\Delta_{i j R}^{m} \geq \delta_{i j R}
$$

where $\delta_{i j R}$ is a positive constant. The term $\Delta_{i j R}^{m}$ is the minimum value of $\Delta_{i j}$ when the agents $i$ and $j$ are within their communication range, i.e.,

$$
\Delta_{i j R}^{m}=\inf \left(\Delta_{i j}\right) \text { s.t. }\left\{\begin{array}{l}
\phi_{i j} \in \mathbb{R}, \\
\bar{x}_{i j}^{2}+\bar{y}_{i j}^{2} \geq \min \left(R_{i}^{2}, R_{j}^{2}\right),
\end{array}\right.
$$

for all $(i, j) \in \mathbb{N}$ and $j \neq i$.

3) The agent $i$ broadcasts $\boldsymbol{q}_{i}$ and $\boldsymbol{q}_{i d}$ in its communication area, and can receive $\boldsymbol{q}_{j}$ and $\boldsymbol{q}_{j d}$ broadcasted by other agents $j, j \in \mathbb{N}, j \neq i$ in the group if the points $O_{j}$ of these agents are in the communication area of the agent $i$.

4) At the initial time $t_{0} \geq 0$, all the agents are sufficiently far away from each other, i.e., the following condition holds:

$$
\Delta_{i j}\left(t_{0}\right) \geq \delta_{i j 0}
$$

where $\delta_{i j 0}$ is a positive constant, and $\Delta_{i j}\left(t_{0}\right)$ is given in (1) evaluated at $\boldsymbol{q}_{i}=\boldsymbol{q}_{i}\left(t_{0}\right)$ and $\boldsymbol{q}_{j}=\boldsymbol{q}_{j}\left(t_{0}\right)$.

Remark 3.1. Items 2) and 3) in Assumption 3.1 specify the way each agent communicates with other agents within its communication range. In Fig. 3, the agents $i$ and $i-1$ are communicating with each other since the points $O_{i-1}$ and $O_{i}$ are in the communication areas of the agents $i$ and $i-1$, respectively. The agents $i$ and $i+1$ are not communicating with each other. In Item 2), the condition (12) holds if there exists a positive constant, $\varrho_{i}$, sufficiently large such that

$$
R_{i} \geq \varrho_{i}+\max \left(a_{i}+a_{j}, b_{i}+b_{j}, a_{i}+b_{j}, a_{j}+b_{i}\right),
$$

for all $(i, j) \in \mathbb{N}$ and $j \neq i$. Moreover, the constant $\Delta_{i j R}^{m}$ is to be determined numerically. In Item 4), we have abused the notation of $\Delta_{i j}\left(x_{i j}\left(t_{0}\right), y_{i j}\left(t_{0}\right), \phi_{i j}\left(t_{0}\right), \phi_{i}\left(t_{0}\right)\right)$ as $\Delta_{i j}\left(t_{0}\right)$ for simplicity of presentation.

Formation Control Objective 3.1. Under Assumption 3.1, for each agent $i$ design $\boldsymbol{u}_{i}$ such that $\boldsymbol{q}_{i}$ tracks $\boldsymbol{q}_{i d}$ while avoids collision with all other agents in the group. In addition, $s_{i d}$ and $\dot{s}_{i d}$ of $\boldsymbol{q}_{i d}$ are to approach the common reference trajectory parameter $s_{\text {od }}$ and its rate $\dot{s}_{o d}$. Specifically, we will design $\boldsymbol{u}_{i}$ such that

$$
\lim _{t \rightarrow \infty} \chi_{i e}(t)=0, \Delta_{i j}(t) \geq \delta_{i j},
$$

where $\chi_{i e}(t)=\left(\left(\boldsymbol{q}_{i}(t)-\boldsymbol{q}_{i d}(t)\right),\left(s_{i d}(t)-s_{\text {od }}(t)\right),\left(\dot{s}_{i d}(t)-\dot{s}_{\text {od }}(t)\right)\right)$, for all $(i, j) \in \mathbb{N}, i \neq j$, and $t \geq t_{0} \geq 0$, where $\delta_{i j}$ is a positive constant.

\section{Formation Control Design}

\subsection{Potential Function}

A potential function consists of the goal function $\gamma$ and the collision avoidance function $\beta$ as follows:

$$
\varphi=\gamma+\beta
$$

where $\gamma$ and $\beta$ are specified below.

The goal function $\gamma$ is designed such that it puts penalty on the tracking errors between the reference and actual trajectories of all the agents. This function is chosen as follows:

$$
\gamma=\sum_{i=1}^{N} \gamma_{i}
$$

where

$$
\gamma_{i}=\frac{k_{1 x}}{2}\left(x_{i}-x_{i d}\right)^{2}+\frac{k_{1 y}}{2}\left(y_{i}-y_{i d}\right)^{2}+\frac{k_{2}}{2}\left(\phi_{i}-\phi_{i d}\right)^{2},
$$

with $k_{1 x}, k_{1 y}$ and $k_{2}$ being positive constants. 
The collision function $\beta$ prevents collision between the agents when they are inside their communication ranges. This function is chosen as follows:

$$
\beta=\sum_{i=1}^{N-1} \sum_{j=i+1}^{N} \beta_{i j}
$$

The function $\beta_{i j}$ is a function of $\Delta_{i j}$, where $\Delta_{i j}$ is given in (1), and has the following properties for all $(i, j) \in \mathbb{N}$ and $i \neq j$ :

1) $\beta_{i j}=0, \beta_{i j}^{\prime}=0, \beta_{i j}^{\prime \prime}=0$ if $\Delta_{i j} \geq \min \left(\delta_{i j d}, \delta_{i j R}\right)$,

2) $\beta_{i j}>0, \beta_{i j}^{\prime}<0$ if $0<\Delta_{i j}<\min \left(\delta_{i j d}, \delta_{i j R}\right)$,

3) $\beta_{i j}=\infty, \beta_{i j}^{\prime}=\infty$ if $\Delta_{i j}=0$,

4) $\beta_{i j} \leq \mu_{1},\left\|\beta_{i j}^{\prime}\right\| \leq \mu_{2},\left\|\beta_{i j}^{\prime \prime}\right\| \leq \mu_{3}$ if $0<\Delta_{i j} \leq \Delta_{i j R}^{M}$,

5) $\beta_{i j}^{\prime}-\left.\beta_{i j}^{\prime}\right|_{\Delta_{i j}=\Delta_{i j c}} \leq-\mu_{4}$ if $0<\Delta_{i j}<\Delta_{i j c} \leq \Delta_{i j R}^{M}$,

6) $\beta_{i j}$ is at least twice differentiable if $\Delta_{i j}>0$,

where $\beta_{i j}^{\prime}=\frac{\partial \beta_{i j}}{\partial \Delta_{i j}} ; \beta_{i j}^{\prime \prime}=\frac{\partial^{2} \beta_{i j}}{\partial \Delta_{i j}^{2}} ; \mu_{1}, \ldots, \mu_{4}$ are positive constants; and $\Delta_{i j R}^{M}$ is the maximum value of $\Delta_{i j}$ when the agents $i$ and $j$, for all $(i, j) \in \mathbb{N}, j \neq j$, are in their communication range, i.e.,

$$
\Delta_{i j R}^{M}=\sup \left(\Delta_{i j}\right) \text { s.t. }\left\{\begin{array}{l}
\phi_{i j} \in \mathbb{R}, \\
\bar{x}_{i j}^{2}+\bar{y}_{i j}^{2} \leq \max \left(R_{i}^{2}, R_{j}^{2}\right) .
\end{array}\right.
$$

Remark 4.1. Properties 1) - 3) imply that the function $\beta$ is positive definite when the agents are in their communication areas, is equal to zero when all the agents are at their desired location, and is equal to infinity when a collision between any agents occurs. Moreover, Property 1) and the function $\gamma$ ensures that the function $\varphi$ attains the (unique) minimum value of zero when all the agents track their reference trajectories perfectly. Properties 3) and 4) are used to prove stability of the closed loop system. Property 5) allows us to use control design and stability analysis methods for continuous systems to handle the collision avoidance problem under the agents' limited communication ranges.

The $p$-times differentiable step function allows us to find many functions that satisfy all properties in (21). As an example, we use the following function $\beta_{i j}$ in the rest of the paper:

$$
\beta_{i j}=\frac{1-h_{i j}\left(\Delta_{i j}, a_{i j}, b_{i j}\right)}{\Delta_{i j}^{2}},
$$

where $h_{i j}\left(\Delta_{i j}, a_{i j}, b_{i j}\right)$ is a $p$-times differentiable step function defined in Definition 2.1 with $p \geq 2$ and $g(y)=y^{p}$. The constants $a_{i j}$ and $b_{i j}$ are chosen such that

$$
0<a_{i j}<b_{i j} \leq \min \left(\delta_{i j d}, \delta_{i j R}\right)-\mu_{i j},
$$

where $\delta_{i j d}$ and $\delta_{i j R}$ are given in (11) and (12), and $\mu_{i j}$ is a positive constant less than $\min \left(\delta_{i j d}, \delta_{i j R}\right)$.

\subsection{Control Design}

Let us define the following vectors

$$
\begin{aligned}
& \boldsymbol{p}_{i}=\left[\begin{array}{ll}
x_{i} & y_{i}
\end{array}\right]^{T}, \boldsymbol{p}_{i j}=\boldsymbol{p}_{i}-\boldsymbol{p}_{j}, \\
& \boldsymbol{p}_{i d}=\left[\begin{array}{ll}
x_{i d} & y_{i d}
\end{array}\right]^{T}, \boldsymbol{p}_{i j d}=\boldsymbol{p}_{i d}-\boldsymbol{p}_{j d},
\end{aligned}
$$

for all $(i, j) \in \mathbb{N}$ and $j \neq i$. With (25), we can write the expression of $\Delta_{i j}$ in (1) as follows:

$$
\Delta_{i j}=0.5\left(\left\|\boldsymbol{Q}_{i j} \boldsymbol{p}_{i j}\right\|^{2}-1\right),
$$

where the matrix $\boldsymbol{Q}_{i j}$ is given by

$$
\boldsymbol{Q}_{i j}=\left[\begin{array}{rr}
\frac{\kappa_{i j} \cos \left(\alpha_{i j}\right)}{a_{i}\left(\kappa_{i j}+\hat{a}_{j}^{2}\right)} & \frac{\kappa_{i j} \sin \left(\alpha_{i j}\right)}{b_{i}\left(\kappa_{i j}+\hat{a}_{j}^{2}\right)} \\
-\frac{\kappa_{i j} \sin \left(\alpha_{i j}\right)}{a_{i}\left(\kappa_{i j}+\hat{b}_{j}^{2}\right)} & \frac{\kappa_{i j} \cos \left(\alpha_{i j}\right)}{b_{i}\left(\kappa_{i j}+\hat{b}_{j}^{2}\right)}
\end{array}\right] \boldsymbol{R}^{-1}\left(\phi_{i}\right) .
$$

With (25), we can see that $\Delta_{i j}$ is a differentiable function of $\boldsymbol{p}_{i j}, \phi_{i j}$ and $\phi_{i}$. Substituting the goal function $\gamma$ with $\gamma_{i}$ given in (19) and the collision function $\beta$ in (20) into (17) results in

$\varphi=\frac{1}{2} \sum_{i=1}^{N}\left(\boldsymbol{p}_{i}-\boldsymbol{p}_{i d}\right)^{T} \boldsymbol{K}_{1}\left(\boldsymbol{p}_{i}-\boldsymbol{p}_{i d}\right)+\frac{k_{2}}{2} \sum_{i=1}^{N}\left(\phi_{i}-\phi_{i d}\right)^{2}+\sum_{i=1}^{N-1} \sum_{j=i+1}^{N} \beta_{i j}$,

where $\boldsymbol{K}_{1}=\operatorname{diag}\left(k_{1 x}, k_{1 y}\right)$. To calculate $\dot{\varphi}$ for designing $\boldsymbol{u}_{i}$, we first calculate $\dot{\kappa}_{i j}$ from (2) as follows:

$$
\dot{\kappa}_{i j}=-\left(\frac{\partial F_{i j}}{\partial \kappa_{i j}}\right)^{-1}\left(\left(\frac{\partial F_{i j}}{\partial \boldsymbol{p}_{i j}}\right)^{T} \dot{\boldsymbol{p}}_{i j}+\frac{\partial F_{i j}}{\partial \phi_{i j}} \dot{\phi}_{i j}+\frac{\partial F_{i j}}{\partial \phi_{i}} \dot{\phi}_{i}\right),
$$

where it is noted that $\frac{\partial F_{i j}}{\partial \kappa_{i j}}$ is always nonzero, see Appendix A, and

$$
F_{i j}=\left(\frac{\hat{a}_{j} \hat{x}_{i j}}{\kappa_{i j}+\hat{a}_{j}^{2}}\right)^{2}+\left(\frac{\hat{b}_{j} \hat{y}_{i j}}{\kappa_{i j}+\hat{b}_{j}^{2}}\right)^{2} .
$$

Now differentiating both sides of (28) along the solutions of (10) and using (29) results in

$$
\begin{aligned}
\dot{\varphi}= & \sum_{i=1}^{N}\left[\boldsymbol{K}_{1}\left(\boldsymbol{p}_{i}-\boldsymbol{p}_{i d}\right)-\sum_{j=1}^{i-1} \beta_{j i}^{\prime} \boldsymbol{G}_{j i} \boldsymbol{p}_{j i}+\sum_{j=i+1}^{N} \beta_{i j}^{\prime} \boldsymbol{G}_{i j} \boldsymbol{p}_{i j}\right]^{T} \\
& \times\left[\begin{array}{c}
u_{x i}-\dot{x}_{i d} \\
u_{y i}-\dot{y}_{i d}
\end{array}\right]+\sum_{i=1}^{N}\left[k_{2}\left(\phi_{i}-\phi_{i d}\right)-\sum_{j=1}^{i-1} \beta_{j i}^{\prime} H_{j i}\right. \\
& \left.+\sum_{j=i+1}^{N}\left(\beta_{i j}^{\prime}\left(H_{i j}+L_{i j}\right)\right)\right]\left(u_{\phi i}-\dot{\phi}_{i d}\right)+\sum_{i=1}^{N} \Phi_{i d} \dot{s}_{i d},
\end{aligned}
$$

where we have used $\dot{\boldsymbol{p}}_{i}-\dot{\boldsymbol{p}}_{j}=\left(\dot{\boldsymbol{p}}_{i}-\dot{\boldsymbol{p}}_{i d}\right)-\left(\dot{\boldsymbol{p}}_{j}-\dot{\boldsymbol{p}}_{j d}\right)+\left(\dot{\boldsymbol{p}}_{i d}-\dot{\boldsymbol{p}}_{j d}\right)$, $\dot{\phi}_{i}=\left(\dot{\phi}_{i}-\dot{\phi}_{i d}\right)+\dot{\phi}_{i d}$, and

$$
\begin{aligned}
\boldsymbol{G}_{i j}= & {\left[\boldsymbol{Q}_{i j}^{T}\left(\boldsymbol{Q}_{i j}-\frac{\partial \boldsymbol{Q}_{i j}}{\partial \kappa_{i j}} \boldsymbol{p}_{i j}\left(\frac{\partial F_{i j}}{\partial \kappa_{i j}}\right)^{-1}\left(\frac{\partial F_{i j}}{\partial \boldsymbol{p}_{i j}}\right)^{T}\right)\right]^{T}, } \\
H_{i j}= & \boldsymbol{p}_{i j}^{T} \boldsymbol{Q}_{i j}^{T}\left(\frac{\partial \boldsymbol{Q}_{i j}}{\partial \phi_{i j}} \boldsymbol{p}_{i j}-\frac{\partial \boldsymbol{Q}_{i j}}{\partial \kappa_{i j}} \boldsymbol{p}_{i j}\left(\frac{\partial F_{i j}}{\partial \kappa_{i j}}\right)^{-1} \frac{\partial F_{i j}}{\partial \phi_{i j}}\right), \\
L_{i j}= & \boldsymbol{p}_{i j}^{T} \boldsymbol{Q}_{i j}^{T}\left(\frac{\partial \boldsymbol{Q}_{i j}}{\partial \phi_{i}} \boldsymbol{p}_{i j}-\frac{\partial \boldsymbol{Q}_{i j}}{\partial \kappa_{i j}} \boldsymbol{p}_{i j}\left(\frac{\partial F_{i j}}{\partial \kappa_{i j}}\right)^{-1} \frac{\partial F_{i j}}{\partial \phi_{i}}\right), \\
\Phi_{i d}= & {\left[-\sum_{j=1}^{i-1} \beta_{j i}^{\prime} \boldsymbol{G}_{j i} \boldsymbol{p}_{j i}+\sum_{j=i+1}^{N} \beta_{i j}^{\prime} \boldsymbol{G}_{i j} \boldsymbol{p}_{i j}\right]^{T}\left[\begin{array}{c}
x_{i d}^{\prime} \\
y_{i d}^{\prime}
\end{array}\right]+} \\
& {\left[-\sum_{j=1}^{i-1} \beta_{j i}^{\prime} H_{j i}+\sum_{j=i+1}^{N} \beta_{i j}^{\prime}\left(H_{i j}+L_{i j}\right)\right] \phi_{i d}^{\prime}, }
\end{aligned}
$$

with $x_{i d}^{\prime}=\frac{\partial x_{i d}}{\partial s_{i d}}, y_{i d}^{\prime}=\frac{\partial y_{i d}}{\partial s_{i d}}$, and $\phi_{i d}^{\prime}=\frac{\partial \phi_{i d}}{\partial s_{i d}}$.

Remark 4.2. A direct calculation of elements of the matrix $\boldsymbol{G}_{i j}$ in (32) from (27) and (30) shows that the matrix $\boldsymbol{G}_{i j}$ is positive definite for all $\boldsymbol{p}_{i j} \in \mathbb{R}^{2}, \phi_{i j} \in \mathbb{R}$ and $\phi_{i} \in \mathbb{R}$ as long as $\Delta_{i j}>0$. The inequality $\Delta_{i j}>0$ is to be guaranteed by our control design later. 
From (31), we choose the control input vector $\boldsymbol{u}_{i}$ as follows:

$$
\begin{aligned}
& {\left[\begin{array}{c}
u_{x i} \\
u_{y i}
\end{array}\right]=-C_{1}\left[\begin{array}{c}
\Omega_{x i} \\
\Omega_{y i}
\end{array}\right]+\left[\begin{array}{c}
\dot{x}_{i d} \\
\dot{y}_{i d}
\end{array}\right],} \\
& u_{\phi i}=-c_{2} \Omega_{\phi i}+\dot{\phi}_{i d},
\end{aligned}
$$

where $\boldsymbol{C}_{1}$ is a $2 \times 2$ positive definite diagonal matrix, $c_{2}$ is a positive constant, and

$$
\begin{aligned}
& {\left[\begin{array}{c}
\Omega_{x i} \\
\Omega_{y i}
\end{array}\right]=\boldsymbol{K}_{1}\left(\boldsymbol{p}_{i}-\boldsymbol{p}_{i d}\right)-\sum_{j=1}^{i-1} \beta_{j i}^{\prime} \boldsymbol{G}_{j i} \boldsymbol{p}_{j i}+\sum_{j=i+1}^{N} \beta_{i j}^{\prime} \boldsymbol{G}_{i j} \boldsymbol{p}_{i j},} \\
& \Omega_{\phi i}=k_{2}\left(\phi_{i}-\phi_{i d}\right)-\sum_{j=1}^{i-1} \beta_{j i}^{\prime} H_{j i}+\sum_{j=i+1}^{N} \beta_{i j}^{\prime}\left(H_{i j}+L_{i j}\right) .
\end{aligned}
$$

However, the term $\Phi_{i d} \dot{s}_{i d}$ in (31) seems to be troublesome because $\dot{\boldsymbol{q}}_{i d}$ is nonzero in general since we are solving the formation tracking control problem. To get around this problem, we will design an update law $\dot{s}_{i d}$ such that $\Phi_{i d} \dot{s}_{i d}=0$ holds for all time and that $s_{i d}$ and $\dot{s}_{i d}$ asymptotically tend to $s_{o d}$ and $\dot{s}_{o d}$. As such, we utilize the $p$-times differentiable step function to design an update law $\dot{\boldsymbol{s}}_{i d}$ as follows:

$$
\dot{s}_{i d}=h\left(\Delta_{i j}, a_{i j d}, b_{i j d}\right)\left(-k_{i d}\left(s_{i d}-s_{o d}\right)+\dot{s}_{o d}\right),
$$

where $k_{i d}$ is a positive constant, and $s_{i d}\left(t_{0}\right)=s_{\text {od }}\left(t_{0}\right)$. The function $h\left(\Delta_{i j}, a_{i j d}, b_{i j d}\right)$ is a $p$-times differentiable step function with $p \geq 1$. The constants $a_{i j d}$ and $b_{i j d}$ are chosen as:

$$
\min \left(\delta_{i j d}, \delta_{i j R}\right)-\mu_{i j} \leq a_{i j d}<b_{i j d}<\min \left(\delta_{i j d}, \delta_{i j R}\right),
$$

where $\delta_{i j d}, \delta_{i j R}$, and $\mu_{i j}$ are given in (11), (12), and (24), respectively. Using properties of the $p$ times differentiable step function, the choice of the constants $a_{i j}, b_{i j}, a_{i j d}$ and $b_{i j d}$ in (24) and (36) results in $h^{\prime}\left(\Delta_{i j}, a_{i j}, b_{i j}\right) h\left(\Delta_{i j}, a_{i j d}, b_{i j d}\right)=0$ and $\left(1-h\left(\Delta_{i j}, a_{i j}, b_{i j}\right)\right) h\left(\Delta_{i j}, a_{i j d}, b_{i j d}\right)=0$. These equalities imply that $\Phi_{i d} \dot{s}_{i d}=0$ holds as long as $\Delta_{i j}>0$, which is to be guaranteed by our control design. Moreover, the choice of the constants $a_{i j d}$ and $b_{i j d}$ in (36) ensures that the function $h\left(\Delta_{i j}, a_{i j d}, b_{i j d}\right)$ approaches 1 whenever $\Delta_{i j}$ approaches $\min \left(\delta_{i j d}, \delta_{i j R}\right)$. This means that $s_{i d}$ and $\dot{s}_{i d}$ are to approach $s_{o d}$ and $\dot{s}_{o d}$ as required. The inequality $\Delta_{i j} \geq \min \left(\delta_{i j d}, \delta_{i j R}\right)>0$ will be guaranteed by our designed control input vector $\boldsymbol{u}_{i}$ in (33). This will be shown in the proof of the main result.

Remark 4.3. 1) The control vector $\boldsymbol{u}_{i}=\left[u_{x i} u_{y i} u_{\phi i}\right]^{T}$ in (33) of the agent $i$ is differentiable and depends on only its own state and reference trajectory, and the states of other agents $j$ in the communication range of the agent $i$ due to Properties 1) and 6) of $\beta_{i j}$ in (21).

2) The update law $\dot{s}_{i d}$ in (35) for $\boldsymbol{q}_{i d}$ ensures that when the collision avoidance is active, the reference trajectory $\boldsymbol{q}_{i d}$ is not updated. This implies that the control law $\boldsymbol{u}_{i}$ gives priority to the collision avoidance mission or the reference trajectory tracking mission whenever which mission is more important.

Substituting the control law $\boldsymbol{u}_{i}$ in (33) and the update law $\dot{\boldsymbol{s}}_{i d}$ in (35) into (31) results in

$$
\dot{\varphi}=-\sum_{i=1}^{N}\left(\left[\Omega_{x i} \Omega_{y i}\right] \boldsymbol{C}_{1}\left[\begin{array}{l}
\Omega_{x i} \\
\Omega_{y i}
\end{array}\right]+c_{2} \Omega_{\phi i}^{2}\right) .
$$

Substituting (33) into (10) results in the closed loop system

$$
\begin{aligned}
& \dot{\boldsymbol{p}}_{i}=-\boldsymbol{C}_{1}\left[\begin{array}{c}
\Omega_{x i} \\
\Omega_{y i}
\end{array}\right]+\left[\begin{array}{c}
\dot{x}_{i d} \\
\dot{y}_{i d}
\end{array}\right], \\
& \dot{\phi}_{i}=-c_{2} \Omega_{\phi i}+\dot{\phi}_{i d},
\end{aligned}
$$

for all $i \in \mathbb{N}$. We now present the main result of our paper.

Theorem 4.1. Under Assumption 3.1, the control input vector $\boldsymbol{u}_{i}=\left[u_{x i} u_{y i} u_{\phi i}\right]^{T}$ in (33) and the update law $\dot{s}_{i d}$ for $\boldsymbol{q}_{i d}$ in (35) for the agent $i$ solve the formation control objective. In particular, no collisions between any agents can occur for all $t \geq t_{0} \geq 0$, the closed loop system (38) is forward complete, and the trajectory $\boldsymbol{q}_{i}$ of the agent $i$ tracks its reference trajectory $\boldsymbol{q}_{\text {id }}$ asymptotically, for all $i=1, \ldots, N$.

\section{Proof. See Appendix B.}

In comparison with the work on formation control of point agents (e.g., Ogren et al. (2004), Tanner and Kumar (2005), Do (2007)), the proposed formation control design for elliptical agents in this paper is substantially involved due to the followings:

1) While a collision avoidance condition for point agents is as simple as positivity of the relative distance between the agents, the condition for collision avoidance between elliptical agents is more complicated. This condition depends on both relative distance between the elliptical agents and orientation of each agent in a complex manner, see Lemma 2.1.

2) While a potential function for formation control design of point agents is a function of only the relative distance between agents, the potential function for designing a formation controller for elliptical agents must be a function of both relative distance between the elliptical agents and orientation of each agent, see (28) with $\beta_{i j}$ being satisfied all the properties listed in (21). Moreover, the relative distance between the agents and orientation of each agent are nonlinearly coupled in the potential function.

3) Nonlinear couplings of the relative distance between the agents and orientation of each agent in the potential function requires a careful design of an update law for the reference trajectory's parameter for each agent, see (35), to make the derivative of the potential function negative definite.

4) Dependence of the potential function for formation control design of elliptical agents on both the relative distance between the agents and orientation of each agent results in more equilibrium points of the resulting closed loop system (38). Therefore, proof of asymptotic stability of only the desired equilibrium point, where the tracking errors are zero, and instability of all other equilibrium points is harder than proof of these results for point agents, see Appendix B.4.

\section{Simulation results}

We use 15 elliptical agents with $a_{i}=4, b_{i}=1$, and $R_{i}=8$. The initial conditions are $\boldsymbol{q}_{i}(0)=\left[R_{o} \sin \left(\frac{2 \pi(i-1)}{R_{o}}+\right.\right.$ $\left.\pi), R_{o} \cos \left(\frac{2 \pi(i-1)}{R_{o}}+\pi\right), 2 \pi+\operatorname{rand}(\bullet)\right]^{T}$ with $R_{o}=10$ for $i=1, \ldots, 5$ and $R_{o}=20$ for $i=6, \ldots, 15$, and $\operatorname{rand}(\bullet)$ a random number between 0 and 1 . The control parameters are chosen as: $\boldsymbol{C}_{1}=10^{4} \operatorname{diag}(1,1), \boldsymbol{K}_{1}=\boldsymbol{C}_{1}^{-1}, c_{2}=10^{4}$, $k_{2}=1 / c_{2}$. We carry out a straight-line formation by choosing $\boldsymbol{q}_{i d}=\left[s_{i d}, \frac{-3 N}{2}+3 i, 0\right]^{T}$ with $s_{i d}(0)=0$, i.e., all the agents are to be put in a vertical stack then move horizontally. We choose $s_{o d}=10 t$ and $\dot{s}_{o d}=10$. The parameters $a_{i j}$ and $b_{i j}$ of $\beta_{i j}$ in (23) are chosen as $a_{i j}=0$ and $b_{i j}=0.5$. The parameters $a_{i j d}$ and $b_{i j d}$ in $h\left(\Delta_{i j}, a_{i j d}, b_{i j d}\right)$, see (35), are chosen as $a_{i j d}=0.6$ and $b_{i j d}=0.85$. A calculation shows that the above initial conditions, $\boldsymbol{q}_{i d}$ and the control parameters satisfy all the conditions (11), (14), (15), (24), and (36). 
Simulation results are plotted in Fig. 4 and Fig. 5. The representative distance $\Delta_{i j}^{*}=\left(\prod_{j \in \mathbb{N} j \neq i} \Delta_{i j}\right)^{1 / 26}$ is plotted in the first sub-figure of Fig.5. The tracking errors $x_{e}=\left[x_{1}-\right.$ $\left.x_{1 d}, \ldots, x_{i}-x_{i d}, \ldots, x_{N}-x_{N d}\right]^{T}, y_{e}=\left[y_{1}-y_{1 d}, \ldots, y_{i}-y_{i d}, \ldots, y_{N}-\right.$ $\left.y_{N d}\right]^{T}$, and $\phi_{e}=\left[\phi_{1}-\phi_{1 d}, \ldots, \phi_{i}-\phi_{i d}, \ldots, \phi_{N}-\phi_{N d}\right]^{T}$ are plotted in the second, third and fourth sub-figure of Fig.5. It is clearly seen from Fig.4 and Fig.5 that there is no collision between any agents as indicated by $\Delta_{i j}^{*}>0$ for all $i=1, \ldots, 15$. Moreover, all the agents track their reference trajectories nicely even though they have to move complicatedly to avoid collide with each other. In Fig.4, several snapshots of the position and orientation of all agents are plotted.
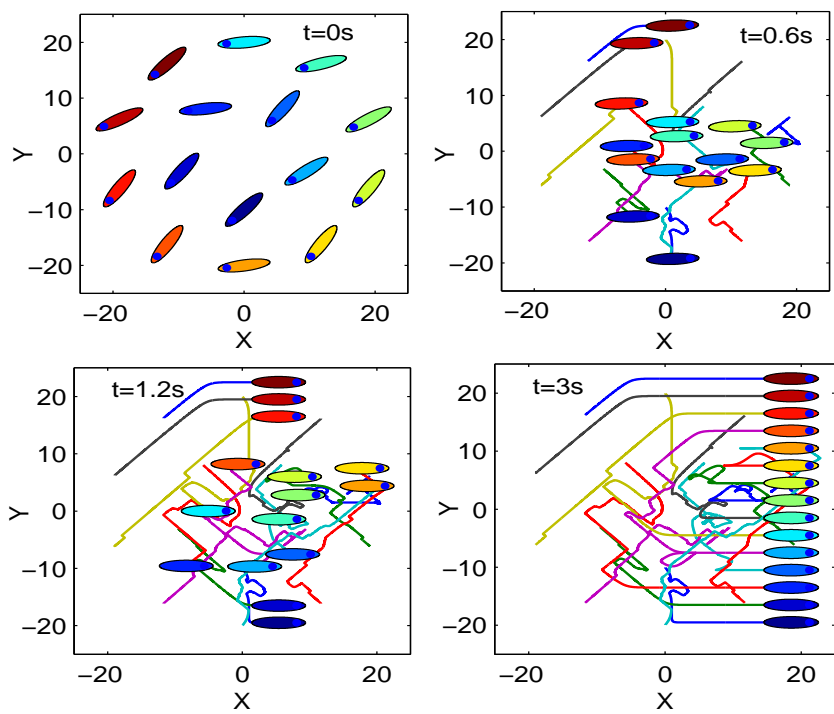

Figure 4: Snapshots of the agents' position and orientation.

\section{Conclusions}

This paper has presented a method to design a formation control system for elliptical agents. The tools used for the success of the formation control design were a separation condition between ellipses, $p$-times differentiable step functions, and potential functions. An extension of the proposed formation control design in this paper and those controllers designed for single underactuated ships in Do and Pan (2009) to provide to a formation control system for a group of underactuated ships is under consideration.

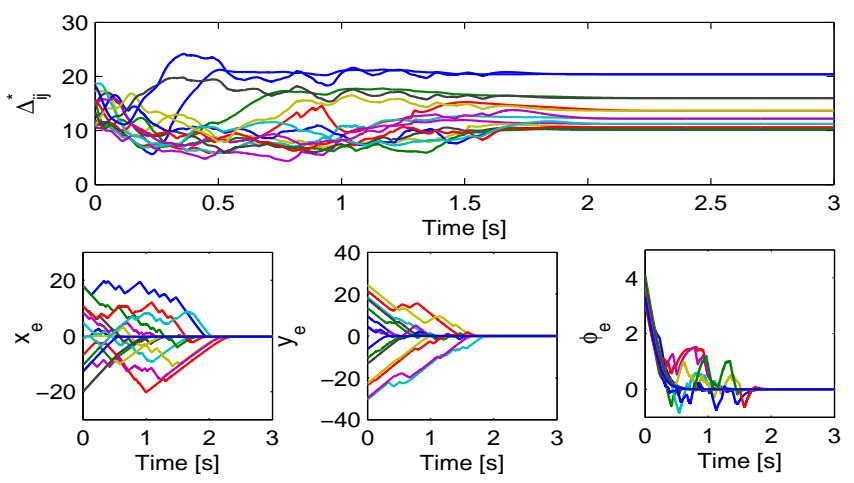

Figure 5: Representative $\Delta_{i j}^{*}$ and tracking errors.

\section{Appendix A. Proof of Lemma 2.1}

From Fig. 2, the boundaries of the disks $i$ and $j$ (equations of points $w_{i b}$ and $w_{j b}$ ) coordinated in the frame $O_{i} X_{i} Y_{i}$ attached to the disk $i$ can be described by

$$
\begin{aligned}
& \mathrm{E}_{i}: x_{i b}^{2} / a_{i}^{2}+y_{i b}^{2} / b_{i}^{2}=1, \\
& \mathrm{E}_{j}:\left[\begin{array}{l}
x_{j b} \\
y_{j b}
\end{array}\right]=\left[\begin{array}{l}
\bar{x}_{i j} \\
\bar{y}_{i j}
\end{array}\right]+\boldsymbol{R}^{-1}\left(\phi_{i j}\right)\left[\begin{array}{c}
a_{j} \cos \left(\theta_{j}\right) \\
b_{j} \sin \left(\theta_{j}\right)
\end{array}\right],
\end{aligned}
$$

where $\bar{x}_{i j}, \bar{y}_{i j}, \phi_{i j}$ are defined in (4), and $\theta_{j} \in[0,2 \pi]$ is an auxiliary angle. The ideas to prove Lemma 2.1 are as follows. We first transform the two ellipses $i$ and $j$ to a circle and an ellipse. We then calculate the distance between these circle and ellipse.

1) Transformation of ellipses: We use the following coordinate transformation:

$$
\begin{aligned}
& {\left[\begin{array}{l}
\hat{x}_{i b} \\
\hat{y}_{i b}
\end{array}\right]=\boldsymbol{R}^{-1}\left(\alpha_{i j}\right)\left[\begin{array}{c}
\left(x_{i b}-\bar{x}_{i j}\right) / a_{i} \\
\left(y_{i b}-\bar{y}_{i j}\right) / b_{i}
\end{array}\right],} \\
& {\left[\begin{array}{c}
\hat{x}_{j b} \\
\hat{y}_{j b}
\end{array}\right]=\boldsymbol{R}^{-1}\left(\alpha_{i j}\right)\left[\begin{array}{c}
\left(x_{j b}-\bar{x}_{i j}\right) / a_{i} \\
\left(y_{j b}-\bar{y}_{i j}\right) / b_{i}
\end{array}\right],}
\end{aligned}
$$

where $\alpha_{i j}$ is given in (3). With the coordinate changes (A.2), the ellipses $i$ and $j$ are transformed to:

$$
\begin{aligned}
& \mathrm{C}_{i}:\left(\hat{x}_{i b}-\hat{x}_{i j}\right)^{2}+\left(\hat{x}_{i b}-\hat{y}_{i j}\right)^{2}=1, \\
& \mathrm{E}_{j}: \hat{x}_{j b}^{2} / \hat{a}_{j}^{2}+\hat{y}_{j b}^{2} / \hat{b}_{j}^{2}=1,
\end{aligned}
$$

where $\hat{x}_{i j}$ and $\hat{y}_{i j}$ are given in (3). Now, the ellipse $E_{i}$ has been transformed to the unit circle $C_{i}$ centered at $\left(\hat{x}_{i j}, \hat{y}_{i j}\right)$ while the ellipse $E_{j}$ has become another ellipse centered at the origin and with semi-axes being $\hat{a}_{j}$ and $\hat{b}_{j}$ given in (3).

2) Distance $\Delta_{i j}$ : We now calculate the distance from the center of the unit circle described by the first equation in (A.3), i.e., from the point $\left(\hat{x}_{i j}, \hat{y}_{i j}\right)$ to the ellipse described by the second equation in (A.3). The closest point $\left(\hat{x}_{j b}, \hat{y}_{j b}\right)$ on the ellipse to the point $\left(\hat{x}_{i j}, \hat{y}_{i j}\right)$ must occur so that $\left(\hat{x}_{i j}-\hat{x}_{j b}, \hat{y}_{i j}-\hat{y}_{j b}\right)$ is normal to the ellipse. An outward pointing ellipse normal is $\frac{1}{2} \nabla\left(\left(\hat{x}_{j b} / \hat{a}_{j}\right)^{2}+\left(\hat{y}_{j b} / \hat{b}_{j}\right)^{2}-1\right)=\left(\hat{x}_{j b} / \hat{a}_{j}^{2}, \hat{y}_{j b} / \hat{b}_{j}^{2}\right)$. Therefore, the orthogonality condition is

$$
\left(\hat{x}_{i j}-\hat{x}_{j b}, \hat{y}_{i j}-\hat{y}_{j b}\right)=\kappa_{i j}\left(\hat{x}_{j b} / \hat{a}_{j}^{2}, \hat{y}_{j b} / \hat{b}_{j}^{2}\right),
$$

where $\kappa_{i j}$ is the largest root of the second equation in (A.3) and (A.4), i.e., the equation (2). We must take $\kappa_{i j}$ as the largest root of the equation (2) so as to have the closest point $\left(\hat{x}_{j b}, \hat{y}_{j b}\right)$ on the ellipse (the second equation of (A.3)) to the point $\left(\hat{x}_{i j}, \hat{y}_{i j}\right)$. We may restrict our attention to $\hat{x}_{i j} \geq 0$ and $\hat{y}_{i j} \geq 0$. Points in other quadrants may be handled by symmetry. Also by symmetry, if $\left(\hat{x}_{i j}, \hat{y}_{i j}\right)$ is in the first quadrant, the closest ellipse point $\left(\hat{x}_{j b}, \hat{y}_{j b}\right)$ is in the first quadrant. As such, we consider $\hat{x}_{i j} \geq 0$ and $\hat{y}_{i j} \geq 0$. From (A.4) we have

$$
\hat{x}_{j b}=\frac{\hat{a}_{j}^{2} \hat{x}_{i j}}{\kappa_{i j}+\hat{a}_{j}^{2}}, \hat{y}_{j b}=\frac{\hat{b}_{j}^{2} \hat{y}_{i j}}{\kappa_{i j}+\hat{b}_{j}^{2}} .
$$

From the symmetry of the ellipse the closest point $\left(\hat{x}_{j b}, \hat{y}_{j b}\right)$ should be in the first quadrant, so we need $\hat{x}_{j b} \geq 0$ and $\hat{y}_{j b} \geq 0$ with a note that $\hat{x}_{i j}$ and $\hat{y}_{i j}$ are not equal to zero simultaneously. These constraints force $\kappa_{i j}>-\hat{a}_{j}^{2}$ and $\kappa_{i j}>-\hat{b}_{j}^{2}$, i.e., the total constraint is $\kappa_{i j}>-\min \left(\hat{a}_{j}^{2}, \hat{b}_{j}^{2}\right)$. Substituting (A.5) into the second equation in (A.3) results in (2). Now the distance from 
the point $\left(\hat{x}_{i j}, \hat{y}_{i j}\right)$ to the closet point $\left(\hat{x}_{j b}, \hat{y}_{j b}\right)$ on the ellipse described by the second equation in (A.3) is $d_{i j}=\sqrt{2 \Delta_{i j}+1}-$ 1 where $\Delta_{i j}$ is given in (1). Therefore, two ellipses $i$ and $j$ are separated if $d_{i j}>0$, i.e., the condition (5) must hold.

3) Domain containing the largest solution of equation (2): We here specify the domain, on which the equation (2) has the largest root. As such, from (2), we define $F\left(\kappa_{i j}\right)=$ $\left(\frac{\hat{a}_{j} \hat{x}_{i j}}{\kappa_{i j}+\hat{a}_{j}^{2}}\right)^{2}+\left(\frac{\hat{b}_{j} \hat{y}_{i j}}{\kappa_{i j}+\hat{b}_{j}^{2}}\right)^{2}-1$. Observing that for any point $\left(\hat{x}_{i j}, \hat{y}_{i j}\right)$ with $\hat{x}_{i j} \geq 0$ and $\hat{y}_{i j} \geq 0$ but $\hat{x}_{i j}$ and $\hat{y}_{i j}$ are not equal to zero simultaneously, we have $\frac{\partial F\left(\kappa_{i j}\right)}{\partial \kappa_{i j}}<0$ and $\frac{\partial^{2} F\left(\kappa_{i j}\right)}{\partial \kappa_{i j}^{2}}>0$ for $\kappa_{i j} \in$ $\left(-\min \left(\hat{a}_{j}^{2}, \hat{b}_{j}^{2}\right), \infty\right)$. Moreover, we note that the limits of $F\left(\kappa_{i j}\right)$ as $\kappa_{i j}$ tends to $-\min \left(\hat{a}_{j}^{2}, \hat{b}_{j}^{2}\right)$ from the right and as $\kappa_{i j}$ tends to $\infty$ are $\lim _{\kappa_{i j} \rightarrow-\min \left(\hat{a}_{j}^{2}, \hat{b}_{j}^{2}\right)^{+}} F\left(\kappa_{i j}\right)=\infty$ and $\lim _{\kappa_{i j} \rightarrow \infty} F\left(\kappa_{i j}\right)=-1$. These limits mean that $F\left(\kappa_{i j}\right)$ is a decreasing function for $\kappa_{i j} \in$ $\left(-\min \left(\hat{a}_{j}^{2}, \hat{b}_{j}^{2}\right), \infty\right)$. Hence, the equation (2) has the largest root on the domain $\left(-\min \left(\hat{a}_{j}^{2}, \hat{b}_{j}^{2}\right), \infty\right)$. Proof of Lemma 2.1 is completed.

\section{Appendix B. Proof of Theorem 4.1}

\section{Appendix B.1. Proof of no collisions}

It is seen from (37) that $\varphi(t) \leq \varphi\left(t_{0}\right), \forall t \geq t_{0} \geq 0$, where $\varphi(t)=\sum_{i=1}^{N} \gamma_{i}(t)+\sum_{i=1}^{N-1} \sum_{j=i+1}^{N} \beta_{i j}(t), \varphi\left(t_{0}\right)$ is $\varphi(t)$ with $t$ replaced by $t_{0}, \gamma_{i}$ and $\beta_{i j}$ are given in (19) and (23), respectively. By Assumption 3.1, and Property 4) of $\beta_{i j}, \varphi\left(t_{0}\right)$ is bounded. Hence, $\varphi(t)$ must be also bounded. As a result, $\beta_{i j}\left(\Delta_{i j}(t)\right)$ must be bounded, i.e., $\Delta_{i j}(t)$ must be larger than some positive constant depending on the initial conditions denoted by $\delta_{i j}$ for all $t \geq t_{0} \geq 0$. This implies from Lemma 2.1 that there are no collisions between any agents for all $t \geq t_{0} \geq 0$. Boundedness of $\varphi(t)$ also implies that of $\left(\boldsymbol{q}_{i}(t)-\boldsymbol{q}_{i d}(t)\right)$ for all $t \geq t_{0} \geq 0$. Therefore, the closed loop system (38) is forward complete.

\section{Appendix B.2. Equilibrium set}

We use Lemma 2 in Do (2007) to find the equilibrium set, which the trajectories of the closed loop system (38) tend to. Integrating both sides of (37) gives $\int_{0}^{\infty} \omega(t) d t \leq \varphi\left(t_{0}\right)$, where $\omega(t)=\sum_{i=1}^{N}\left(\left[\Omega_{x i}(t) \Omega_{y i}(t)\right] \boldsymbol{C}_{1}\left[\begin{array}{l}\Omega_{x i}(t) \\ \Omega_{y i}(t)\end{array}\right]+c_{2} \Omega_{\phi i}^{2}(t)\right) . \quad$ The function $\omega(t)$ is scalar, nonnegative and differentiable. The derivative of $\omega(t)$ along the solutions of the closed loop system (38) using Properties 2) and 5) of the function $\beta_{i j}$ in (21) satisfies $\left|\frac{\mathrm{d} \omega(t)}{\mathrm{d} t}\right| \leq M \omega(t)$ with $M$ a positive constant. Therefore Lemma 2 in Do (2007) results in $\lim _{t \rightarrow \infty} \omega(t)=0$, which means from the expression of $\omega(t)$ that

$$
\lim _{t \rightarrow \infty}\left(\Omega_{x i}(t), \Omega_{y i}(t), \Omega_{\phi i}(t)\right)=0 .
$$

Hence the trajectory $\boldsymbol{q}_{i}$ of the agent $i$ asymptotically converges to the equilibrium set, $\boldsymbol{\Xi}$, in which $\Omega_{x i}(t)=0$, $\Omega_{y i}(t)=0$, and $\Omega_{\phi i}(t)=0$. From the expression of $\Omega_{x i}(t)$, $\Omega_{y i}(t)$, and $\Omega_{\phi i}(t)$ in (34), the limits (B.1) imply that $\boldsymbol{q}(t)=$ $\left[\boldsymbol{q}_{1}^{T}(t) \boldsymbol{q}_{2}^{T}(t), \ldots, \boldsymbol{q}_{N}^{T}(t)\right]^{T}$ can tend to $\boldsymbol{q}_{d}=\left[\boldsymbol{q}_{1 d}^{T} \boldsymbol{q}_{2 d}^{T}, \ldots, \boldsymbol{q}_{N d}^{T}\right]^{T}$ with $\boldsymbol{q}_{i d}=\left[x_{i d} y_{i d} \phi_{i d}\right]^{T}$, since $\beta_{i j}^{\prime}(t)=0$ at $\boldsymbol{q}_{i}=\boldsymbol{q}_{i d}$ and $\boldsymbol{q}_{j}=\boldsymbol{q}_{j d}$, for all $(i, j) \in \mathbb{N}$ and $i \neq j$, (see Property 1 ) of $\left.\beta_{i j}\right)$, or tend to a vector denoted by $\boldsymbol{q}_{c}=\left[\boldsymbol{q}_{1 c}^{T} \boldsymbol{q}_{2 c}^{T}, \ldots, \boldsymbol{q}_{N c}^{T}\right]^{T}$ with $\boldsymbol{q}_{i c}=\left[x_{i c} y_{i c} \phi_{i c}\right]^{T}$ as the time goes to infinity, i.e., the equilibrium points can be $\boldsymbol{q}_{d}$ or $\boldsymbol{q}_{c}$. The vector $\boldsymbol{q}_{c}$ is such that

$$
\Omega_{x i c}=0, \Omega_{y i c}=0, \Omega_{\phi i c}=0,
$$

where $\Omega_{x i c}=\left.\Omega_{x i}\right|_{\boldsymbol{q}=\boldsymbol{q}_{c}}, \Omega_{y i c}=\left.\Omega_{y i}\right|_{\boldsymbol{q}=\boldsymbol{q}_{c}}$, and $\Omega_{\phi i c}=\left.\Omega_{\phi i}\right|_{\boldsymbol{q}=\boldsymbol{q}_{c}}$, for all $i \in \mathbb{N}$. Since we have already proved that the trajectory $\boldsymbol{q}$ can approach either the set of desired equilibrium points denoted by $\boldsymbol{q}_{d}$ or the set of undesired equilibrium points denoted by $\boldsymbol{q}_{c}$ 'almost globally'. The term 'almost globally' refers to the fact that the agents start from a set that includes the condition (14) and that does not coincide at any point with the set of the undesired point $\boldsymbol{q}_{c}$. Hence, we need to prove that $\boldsymbol{q}_{\boldsymbol{d}}$ is locally asymptotically stable and that $\boldsymbol{q}_{c}$ is locally unstable.

\section{Appendix B.3. Proof of $\boldsymbol{q}_{d}$ being asymptotically stable}

Linearizing the closed loop system (38) near $\boldsymbol{q}_{d}$ gives

$$
\begin{aligned}
& \dot{\boldsymbol{p}}_{i}=-\boldsymbol{C}_{1}\left(\boldsymbol{K}_{1}\left(\boldsymbol{p}_{i}-\boldsymbol{p}_{i d}\right)\right)+\dot{\boldsymbol{p}}_{i d}, \\
& \dot{\phi}_{i}=-c_{2}\left(k_{2}\left(\phi_{i}(t)-\phi_{i d}(t)\right)\right)+\dot{\phi}_{i d},
\end{aligned}
$$

for all $i \in \mathbb{N}$, where we have used $\left.\beta_{i j}^{\prime}\right|_{\boldsymbol{q}=\boldsymbol{q}_{d}}=0$ and $\left.\beta_{i j}^{\prime \prime}\right|_{\boldsymbol{q}=\boldsymbol{q}_{d}}=0$, see Property 1 ) of the function $\beta_{i j}$ in (21). Local asymptotic stability of the equilibrium $\boldsymbol{q}_{\boldsymbol{d}}$ follows from (B.3) since the first time derivative of the function $V_{d}=\frac{1}{2} \sum_{i=1}^{N}\left[\left(\boldsymbol{p}_{i}-\right.\right.$ $\left.\left.\boldsymbol{p}_{i d}\right)^{T}\left(\boldsymbol{p}_{i}-\boldsymbol{p}_{i d}\right)+\left(\phi_{i}-\phi_{i d}\right)^{2}\right]$ along the solutions of (B.3) satisfies $\dot{V}_{d} \leq-2 \min \left(\lambda_{\min }\left(\boldsymbol{C}_{1} \boldsymbol{K}_{1}\right), c_{2}\right) V_{d}$, where $\lambda_{\min }\left(\boldsymbol{C}_{1} \boldsymbol{K}_{1}\right)$ is the minimum eigenvalue of $\boldsymbol{C}_{1} \boldsymbol{K}_{1}$.

\section{Appendix B.4. Proof of $\boldsymbol{q}_{c}$ being unstable}

Substituting (B.2) into the closed loop system (38) gives

$$
\begin{aligned}
& \dot{\boldsymbol{p}}_{i}=-\boldsymbol{C}_{1}\left(\left[\begin{array}{c}
\Omega_{x i} \\
\Omega_{y i}
\end{array}\right]-\left[\begin{array}{c}
\Omega_{x i c} \\
\Omega_{y i c}
\end{array}\right]\right)+\dot{\boldsymbol{p}}_{i d}, \\
& \dot{\phi}_{i}=-c_{2}\left(\Omega_{\phi i}-\Omega_{\phi i c}\right)+\dot{\phi}_{i d},
\end{aligned}
$$

for all $i \in \mathbb{N}$. We now investigate stability of (B.4) at $\boldsymbol{q}_{c}$.

Let $\mathbb{N}^{*}$ be the set of the agents such that if the agents $i$ and $j$ belong to the set $\mathbb{N}^{*}$ then $\Delta_{i j}\left(\boldsymbol{p}_{i j}, \phi_{i j}, \phi_{i}\right)<\min \left(\delta_{i j d}, \delta_{i j R}\right)$, and $N^{*}$ be the size of the set $\mathbb{N}^{*}$. For those agents in the set $\mathbb{N}^{*}$, the collision avoidance is active. Therefore, $\dot{\boldsymbol{q}}_{i d}=0$, for all $i \in \mathbb{N}^{*}$, see Item 2) in Remark 4.3. Now, from (B.2) we have $\sum_{(i, j) \in \mathbb{N}^{*}} \boldsymbol{p}_{i j c}^{T}\left[\Omega_{x i c}, \Omega_{y i c}\right]^{T}=0$, which can be expanded using (B.2) and (34) as follows:

$$
\sum_{(i, j) \in \mathbb{N}^{*}} \boldsymbol{p}_{i j c}^{T}\left(\boldsymbol{K}_{1}+N^{*} \beta_{i j c}^{\prime} \boldsymbol{G}_{i j c}\right) \boldsymbol{p}_{i j c}=\sum_{(i, j) \in \mathbb{N}^{*}} \boldsymbol{p}_{i j c}^{T} \boldsymbol{K}_{1} \boldsymbol{p}_{i j d} .
$$

Since we have proved that $\left\|\boldsymbol{p}_{i j c}\right\|$ is bounded and $\left\|\boldsymbol{p}_{i j d}\right\|$ is bounded by assumption, (B.5) indicates that $\lim _{\lambda_{\max }\left(\boldsymbol{K}_{1}\right) \rightarrow 0}\left(\left\|\boldsymbol{Q}_{i j c} \boldsymbol{p}_{i j c}\right\|\right)=\infty$ with $\lambda_{\max }\left(\boldsymbol{K}_{1}\right)$ the maximum eigenvalue of $\boldsymbol{K}_{1}$. This means that we can choose a control gain matrix $\boldsymbol{K}_{1}$ such that the matrix $\boldsymbol{K}_{1}+N^{*} \beta_{i j c}^{\prime} \boldsymbol{G}_{i j c}$ is negative definite for some $(i, j)$ with $i \neq j$. Let $\mathbb{N}^{* *} \subset \mathbb{N}^{*}$ be a nonempty set such that for all $(i, j) \in \mathbb{N}^{* *}, i \neq j$, the matrix $\boldsymbol{K}_{1}+N^{*} \beta_{i j c}^{\prime} \boldsymbol{G}_{i j c}$ is negative definite. Since $\mathbb{N}^{* *} \subset \mathbb{N}^{*}$, we have $\dot{\boldsymbol{q}}_{i d}=0$, for all $i \in \mathbb{N}^{* *}$.

To investigate stability of (B.4) at $\boldsymbol{q}_{c}$, we consider the following function for the agents belong to $\mathbb{N}^{* *}$ :

$$
\bar{V}_{c}^{* *}=\frac{1}{2} \sum_{(i, j) \in \mathbb{N}^{* *}}\left(\boldsymbol{p}_{i j}-\boldsymbol{p}_{i j c}\right)^{T} \boldsymbol{C}_{1}^{-1}\left(\boldsymbol{p}_{i j}-\boldsymbol{p}_{i j c}\right)+\frac{1}{2 c_{2}} \sum_{i=1}^{N^{* *}}\left(\phi_{i}-\phi_{i c}\right)^{2}
$$

whose derivative along the solutions of (B.4) is

$$
\dot{\bar{V}}_{c}^{* *}=-\sum_{(i, j) \in \mathbb{N}^{* *}} W_{i j c}-\sum_{i=1}^{N^{* *}} U_{1 i}-N^{* *} \sum_{(i, j) \in \mathbb{N}^{* *}} U_{2 i j},
$$


where

$$
\begin{gathered}
W_{i j c}=\left(\boldsymbol{p}_{i j}-\boldsymbol{p}_{i j c}\right)^{T}\left(\boldsymbol{K}_{1}+N^{* *} \beta_{i j c}^{\prime} \boldsymbol{G}_{i j c}\right)\left(\boldsymbol{p}_{i j}-\boldsymbol{p}_{i j c}\right), \\
U_{1 i}=\left(\phi_{i}-\phi_{i c}\right)\left[k_{2}\left(\phi_{i}-\phi_{i c}\right)-\sum_{j=1}^{i-1} \beta_{j i}^{\prime} H_{j i}+\sum_{j=i+1}^{N^{* *}} \beta_{i j}^{\prime} \times\right. \\
\left.\left(H_{i j}+L_{i j}\right)-\left(-\sum_{j=1}^{i-1} \beta_{j i c}^{\prime} H_{j i c}+\sum_{j=i+1}^{N^{* *}} \beta_{i j c}^{\prime}\left(H_{i j c}+L_{i j c}\right)\right)\right], \\
U_{2 i j}=\left(\boldsymbol{p}_{i j}-\boldsymbol{p}_{i j c}\right)^{T}\left(\beta_{i j}^{\prime} \boldsymbol{G}_{i j}-\beta_{i j c}^{\prime} \boldsymbol{G}_{i j c}\right) \boldsymbol{p}_{i j} \\
\text { with } \beta_{i j c}^{\prime}=\left.\beta_{i j}^{\prime}\right|_{\boldsymbol{q}=\boldsymbol{q}_{c}}, \boldsymbol{G}_{i j c}=\left.\boldsymbol{G}_{i j}\right|_{\boldsymbol{q}=\boldsymbol{q}_{c}}, \boldsymbol{p}_{i j c}=\left.\boldsymbol{p}_{i j}\right|_{\boldsymbol{q}=\boldsymbol{q}_{c}}, H_{i j c}^{\prime}= \\
\left.H_{i j}^{\prime}\right|_{\boldsymbol{q}=\boldsymbol{q}_{c}} \text {, and } L_{i j c}^{\prime}=\left.L_{i j}^{\prime}\right|_{\boldsymbol{q}=\boldsymbol{q}_{c}} \cdot \text { We now define a set } \Psi \text { such that } \\
\Psi=\left\{\left(\boldsymbol{p}_{i j}, \phi_{i}\right) \in B_{r} \mid U_{1} \leq 0 \text { and } U_{2} \leq 0, \forall(i, j) \in \mathbb{N}^{* *}, i \neq j,\right.
\end{gathered}
$$

where $U_{1}=\sum_{i=1}^{N^{* *}} U_{1 i}$ and $U_{2}=\sum_{(i, j) \in \mathbb{N}^{* *}} U_{2 i j}$.

In this set, we have

$$
\dot{\bar{V}}_{c}^{* *} \geq-\sum_{(i, j) \in \mathbb{N}^{* *}} W_{i j c} .
$$

We need to show that the set $\Psi$ is nonempty. For the condition $U_{1 i} \leq 0$, we can always find $\phi_{i}$ as a function of $\boldsymbol{q}_{i c}, \boldsymbol{p}_{i j}$, $\phi_{i j}$ and $\phi_{i}$ for all $(i, j) \in \mathbb{N}^{* *}$ and $i \neq j$ such that $U_{1 i} \leq 0$. An example is $\phi_{i}=\phi_{i c}$ for all $i \in \mathbb{N}^{* *}$. For the condition $U_{2 i j} \leq 0$, we first note that the matrix $\boldsymbol{G}_{i j}$ is positive definite for all $\boldsymbol{p}_{i j} \in \mathbb{R}^{2}, \phi_{i j} \in \mathbb{R}$ and $\phi_{i} \in \mathbb{R}$ such that $\Delta_{i j}>0$, for all $(i, j) \in \mathbb{N}^{* *}$ and $i \neq j$, see Remark 4.2. Second, we note from Properties 2) and 3) of the function $\beta_{i j}$ in (21) that $\beta_{i j}^{\prime}$ is negative and is equal to infinity when $\Delta_{i j}=0$.

Now, let us choose a contact point $\hat{O}_{i j b}$, see Fig. B.6, between the agent $i$ and the agent $j$ belonging to the set $\mathbb{N}^{* *}$, i.e., the point where $\Delta_{i j}=0$ such that the distance from $\hat{O}_{i j b}$ to the point $\hat{O}_{i j c}$ at $\left(\hat{X}_{i j c}, \hat{Y}_{i j c}\right)$ where $\left(\hat{X}_{i j c}, \hat{Y}_{i j c}\right):=\boldsymbol{Q}_{i j c} \boldsymbol{p}_{i j c}$ is smallest. In Fig. B.6, the $\hat{O}_{i j} \hat{X}_{i j}$ and $\hat{O}_{i j} \hat{Y}_{i j}$ axes represents the first and second elements of $\boldsymbol{Q}_{i j} \boldsymbol{p}_{i j}$, respec-

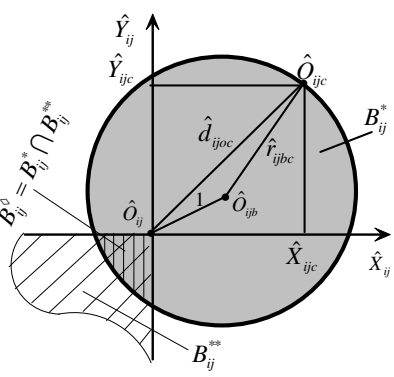

Figure B.6: An unstable set. tively. Let the ball $B_{i j}^{*}$ be centered at $\hat{O}_{i j b}$ and have the radius $\hat{r}_{i j b c}$ of the distance from the center $\hat{O}_{i j b}$ to the point $\hat{O}_{i j c}$. By construction, if $\left(\boldsymbol{p}_{i j}, \phi_{i}\right) \in B_{i j}^{*}$ with $\phi_{i}$ being satisfied the condition $U_{1 i} \leq 0$, the matrix $\boldsymbol{S}_{i j}:=\left(\boldsymbol{Q}_{i j}^{-T}\left(\beta_{i j}^{\prime} \boldsymbol{G}_{i j}-\beta_{i j c}^{\prime} \boldsymbol{G}_{i j c}\right) \boldsymbol{Q}_{i j}^{-1}\right)$ is negative definite. On the other hand, let $B_{i j}^{* *}$ be the set such that if $\left(\boldsymbol{p}_{i j}, \phi_{i}\right) \in B_{i j}^{* *}$ then $\left[\boldsymbol{Q}_{i j}\left(\boldsymbol{p}_{i j}-\boldsymbol{p}_{i j c}\right)\right]^{T} \boldsymbol{Q}_{i j} \boldsymbol{p}_{i j}$ is positive, see Fig. B.6 for an illustration. Let $B_{i j}^{\diamond}=B_{i j}^{*} \cap B_{i j}^{* *}$. We can see that $B_{i j}^{\diamond}$ is nonempty if the radius $\hat{r}_{i j b c}$ is greater than 1 because the distance between the point $\hat{O}_{i j}$ and the point $\hat{O}_{i j b}$ equals 1 . In order to have the radius $\hat{r}_{i j b c}>1$, we need the distance $\hat{d}_{i j o c}$ from the point $\hat{O}_{i j}$ and the point $\hat{O}_{i j c}$ larger than 2.

We now show that $\hat{d}_{i j o c}>2$ by choosing $\boldsymbol{K}_{1}$ with $\lambda_{\max }\left(\boldsymbol{K}_{1}\right)$ sufficiently small. As such, from (B.2) we can again see that $\lim _{\lambda_{\max }\left(\boldsymbol{K}_{1}\right) \rightarrow 0}\left(\left\|\boldsymbol{Q}_{i j c} \boldsymbol{p}_{i j c}\right\|\right)=\infty$. This means that the set $B_{i j}^{\diamond}$ is nonempty for an appropriate $\boldsymbol{K}_{1}$. Since the matrix $\boldsymbol{S}_{i j}$ is negative definite for $\left(\boldsymbol{p}_{i j}, \phi_{i}\right) \in B_{i j}^{*}$ with $\phi_{i}$ being satisfied the condition $U_{1 i} \leq 0$, there exists a nonempty subset $B_{i j}^{\diamond \diamond}$ of $B_{i j}^{\diamond}$ such that if $\left(\boldsymbol{p}_{i j}, \phi_{i}\right) \in B_{i j}^{\diamond \diamond}$ with $\phi_{i}$ being satisfied the condition $U_{1 i} \leq 0$ the condition $U_{2 i j} \leq 0$ holds. Hence, the set $\Psi$ is nonempty and given by $\Psi=\bigcap_{(i, j) \in \mathbb{N}^{* *}} B_{i j}^{\diamond \diamond}$. Since we have already proved that the matrix $\boldsymbol{K}_{1}+N^{*} \beta_{i j c}^{\prime} \boldsymbol{G}_{i j c}$ is negative definite, the function $\bar{V}_{c}^{* *}$ in (B.6), its derivative $\dot{\bar{V}}_{c}^{* *}$ in (B.10) together with the nonempty set $\Psi$ imply that $\boldsymbol{q}_{c}$ is unstable by Chetaev's Theorem (Theorem 4.3 in Khalil (2002)).

\section{References}

Balch, T., Arkin, R.C., 1998. Behavior-based formation control for multirobot teams. IEEE Transactions on Robotics and Automation 14, 926-939.

Choi, Y., W.Wang, Liu, Y., Kim, M., 2006. Continuous collision detection for two moving elliptic disks. IEEE Transactions on Robotics 22, 213-224.

Das, A., Fierro, R., Kumar, V., Ostrowski, J., Spletzer, J., Taylor, C., 2002. A vision based formation control framework. IEEE Transactions on Robotics and Automation 18, 813-825.

Dimarogonas, D.V., Loizou, S.G., Kyriakopoulos, K.J., Zavlanos, M.M. 2006. A feedback stabilization and collision avoidance scheme for multiple independent non-point agents. Automatica 42, 229-243.

Do, K.D., 2007. Bounded controllers for formation stabilization of mobile agents with limited sensing ranges. IEEE Transactions on Automatic Control 52, 569-576.

Do, K.D., 2009. Output-feedback formation tracking control of unicycle-type mobile robots with limited sensing ranges. Robotics and Autonomous Systems 57, 34-47.

Do, K.D., 2010. Practical control of underactuated ships. Ocean Engineering 37, 1111-1119.

Do, K.D., 2011. Formation control of underactuated ships with elliptical shape approximation and limited communication ranges. Automatica, Submitted .

Do, K.D., Pan, J., 2009. Control of Ships and Underwater Vehicles: Design for Underactuated and Nonlinear Marine Systems. Springer.

Egerstedt, M., Hu, X., 2001. Formation constrained multiagent control. IEEE Transactions on Robotics and Austomation 17, 947-951.

Hu, J., Feng, G., 2010. Distributed tracking control of leader follower multiagent systems under noisy measurement. Automatica 46, 1382-1387.

Hussein, I., Bloch, A., 2008. Optimal control of underactuated nonholonomic mechanical systems. IEEE Transactions on Automatic Control 53, 668681.

Hussein, I., Stipanovic, D., 2007. Effective coverage control for mobile sensor networks with guaranteed collision avoidance. IEEE Transactions on Control Systems Technology 15, 642-657.

Jonathan, R.T., Beard, R.W., Young, B., 2003. A decentralized approach to formation maneuvers. IEEE Transactions on Robotics and Automation 19, 933-941.

Khalil, H., 2002. Nonlinear Systems. Prentice Hall.

Ogren, P., Fiorelli, E., Leonard, N.E., 2004. Cooperative control of mobile sensor networks: Adaptive gradient climbing in a distributed environment. IEEE Transactions on Automatic Control 49, 1292-1302.

Olfati-Saber, R., 2006. Flocking for multi-agent dynamic systems: algorithms and theory. IEEE Transactions on Automatic Control 51, 401-420.

Rimon, E., Koditschek, D.E., 1992. Exact robot navigation using artificial potential functions. IEEE Trans. Robot. and Automat. 8, 501-518.

Stipanovic, D.M., Inalhan, G., Teo, R., Tomlin, C.J., 2004. Decentralized overlapping control of a formation of unmanned aerial vehicles. Automatica 40, 1285-1296.

Tanner, H.G., Kumar, A., 2005. Towards decentralization of multi-robot navigation functions, in: Proceedings of the 2005 IEEE International Conference on Robotics and Automation, Barcelona, Spain. pp. 4132-4137.

Wang, P., 1991. Navigation strategies for multiple autonomous mobile robots moving in formation. Journal of Robotic Systems 8, 177-195. 